\title{
A Note on Reverse Minkowski Inequality via Generalized Proportional Fractional Integral Operator with respect to Another Function
}

\author{
Saima Rashid ${ }^{\mathbb{D}},{ }^{1}$ Fahd Jarad, ${ }^{2}$ and Yu-Ming Chu $\mathbb{D}^{3,4}$ \\ ${ }^{1}$ Department of Mathematics, Government College (GC) University, Faisalabad 38000, Pakistan \\ ${ }^{2}$ Department of Mathematics, Çankaya University, Ankara 06530, Turkey \\ ${ }^{3}$ Department of Mathematics, Huzhou University, Huzhou 313000, China \\ ${ }^{4}$ School of Mathematics and Statistics, Changsha University of Science \& Technology, Changsha 410114, China
}

Correspondence should be addressed to Yu-Ming Chu; chuyuming@zjhu.edu.cn

Received 6 November 2019; Accepted 16 March 2020; Published 27 April 2020

Academic Editor: Vincenzo Vespri

Copyright (C) 2020 Saima Rashid et al. This is an open access article distributed under the Creative Commons Attribution License, which permits unrestricted use, distribution, and reproduction in any medium, provided the original work is properly cited.

This study reveals new fractional behavior of Minkowski inequality and several other related generalizations in the frame of the newly proposed fractional operators. For this, an efficient technique called generalized proportional fractional integral operator with respect to another function $\Phi$ is introduced. This strategy usually arises as a description of the exponential functions in their kernels in terms of another function $\Phi$. The prime purpose of this study is to provide a new fractional technique, which need not use small parameters for finding the approximate solution of fractional coupled systems and eliminate linearization and unrealistic factors. Numerical results represent that the proposed technique is efficient, reliable, and easy to use for a large variety of physical systems. This study shows that a more general proportional fractional operator is very accurate and effective for analysis of the nonlinear behavior of boundary value problems. This study also states that our findings are more convenient and efficient than other available results.

\section{Introduction}

Recently, the idea of nonlocal operators of differentiation has boarded out numerous analysts from practically all parts of sciences and engineering due to their abilities to include progressively complex characteristics into numerical conditions. Fractional calculus has also been comprehensively utilized in several instances, but the concept has been popularized and implemented in numerous disciplines of science, technology, and engineering as a mathematical model $[1,2]$. Numerous distinguished generalized fractional integral operators consist of the Hadamard operator, Erdélyi-Kober operators, the Saigo operator, the Gaussian hypergeometric operator, the Marichev-Saigo-Maeda fractional integral operators, and so on, out of the which, the Riemann-Liouville fractional integral operator has been extensively utilized by researchers in theory as well as applications. For added information related to fractional calculus operators and their usefulness, one may also communicate to the expositions by Miller and Ross [3], Samko et al. [4], Kiryakova [5], and Baleanu et al. [6]. Almeida [7] proposed a new fractional derivative called Caputo derivative with respect to another function $\Phi$, and Kilbas et al. [8] explored the concept of Riemann-Liouville fractional integrals with respect to another function $\Phi$.

Within the structure of applied science and mathematical modeling, there exists an outstanding kind of operator known as generalized proportional fractional integral operator with respect to another function $\Phi$ in which the variable is a scaled according to proportionality index $\sigma$. This diversified operator was introduced by Rashid et al. [9], to conceivably role those physical problems for which classical physical law, for example, the well-known Mellin transform, Fourier transform, and probability theory, is suitable; such 
physical issue is accepted to be founded on the fractional calculus and pertinent to the media of nonintegral fractional operators. Amongst others, we estimate real-world issues such as Porous media, aquifer, and turbulence; furthermore, progressively, other media regularly show fractional properties [10-22].

During the most recent decade, integral inequalities have been expanding enthusiasm to employ fractional techniques that have capacious significance to many fields, including neural networks, remote sensing, optimization of structures, optimization of electromagnetic systems, and many other applied sciences [23-33]. Lately, much consideration has been given to the fractional calculus of integral inequalities. We comment that fractional calculus is imperative for a few reasons. We contemplate the subjective conduct of the solution of the integral-differential and difference equations when the given operator and the feasible variations occur in a parameter. Several integral inequalities and their modifications have been derived via the classical fractional operators [34-42].

The first fractional technique was employed on reverse Minkowski inequality in [43]. Lately, Anber et al. [44] proposed some fractional integral inequalities within the scope of Riemann-Liouville fractional integral. In [45], the researchers explored some Minkowski inequalities and other variants by contemplating Katugampola's fractional techniques. In [46-48], many researchers have been focused on their attentions in order to find the distinguished version of the reverse Minkowski inequality for generalized $k$-fractional conformable integral, by generalized proportional fractional integral operator and Hadamard fractional integral operators.

The aim to deal with new operators of integration has been introduced in this paper comprising exponential functions in their kernels in terms of another function $\Phi$ and generalized some well-known fractional operators as generalized proportional fractional integral operator, Riemann-Liouville fractional integral operator, Katugampola fractional integrals, and Hadamard fractional integral operators. The new operators will be referred to as the generalized proportional fractional integral operator with respect to another function $\Phi$. The new operators are expected to fascinate the reverse Minkowski inequality and other associated integral inequalities in the light of a generalized proportional fractional integral operator. Moreover, the numerical approximation of these new operators are additionally given a few utilities to a real-world problem.

\section{Preliminaries}

This segment is dedicated to some recognized definitions and outcomes associated with the generalized conformable fractional integral operators and their generalization related to the generalized conformable fractional integral operators. Set et al., in [49], launched the fractional version of the Hermite-Hadamard and reverse Minkowski inequality. Additionally, Hardy's type and reverse Minkowski inequalities were supplied by Bougoffa in [36]. The subsequent consequences concerning the reverse Minkowski inequalities are the inducement of labor finished to date, concerning the classical integrals.

Theorem 1 (see [49]). Let $v \geq 1, \Upsilon \geq \gamma>0, y>x \geq 0$, and $G$ and $H$ be two positive functions defined on $[0, \infty)$ such that $\gamma \leq(G(z) / H(z)) \leq \Upsilon$, for all $z \in[x, y]$. Then, one has

$$
\begin{aligned}
& \left(\int_{x}^{y} G^{v}(\varphi) \mathrm{d} \varphi\right)^{(1 / v)}+\left(\int_{x}^{y} H^{v}(\varphi) \mathrm{d} \varphi\right)^{(1 / v)} \\
& \quad \leq \frac{1+\Upsilon(\gamma+2)}{(\Upsilon+1)(\gamma+1)}\left(\int_{x}^{y}(G+H)^{v}(\varphi) \mathrm{d} \varphi\right)^{(1 / v)} .
\end{aligned}
$$

Theorem 2 (see [49]). Let $v \geq 1, Y \geq \gamma>0, y>x \geq 0$, and $G$ and $H$ be two positive functions defined on $[0, \infty)$ such that $\gamma \leq(G(z) / H(z)) \leq \Upsilon$, for all $z \in[x, y]$. Then, the inequality

$$
\begin{aligned}
& \left(\int_{x}^{y} G^{v}(\varphi) \mathrm{d} \varphi\right)^{(2 / v)}+\left(\int_{x}^{y} H^{v}(\varphi) \mathrm{d} \varphi\right)^{(2 / v)} \\
& \quad \geq\left(\frac{(1+\gamma)(\Upsilon+1)}{\Upsilon}-2\right)\left(\int_{x}^{y} G^{v}(\varphi) \mathrm{d} \varphi\right)^{(1 / v)}\left(\int_{x}^{y} H^{v}(\varphi) \mathrm{d} \varphi\right)^{(1 / v)},
\end{aligned}
$$

holds.

In [43], Dahmani used the Riemann-Liouville fractional integral operators to prove the subsequent reverse Minkowski inequalities.

Theorem 3 (see [43]). Let $\delta>0, v \geq 1, y>x \geq 0, \Upsilon \geq \gamma>0$, and $G$ and $H$ be two positive functions defined on $[0, \infty)$ such that $\mathscr{T}_{x^{+}}^{\delta} G^{v}(\varphi)<\infty$ and $\mathscr{T}_{x^{+}}^{\delta} H^{v}(\varphi)<\infty$, for all $\varphi>0$. Then, the inequality

$$
\begin{aligned}
& \left(\mathscr{T}_{x^{+}}^{\delta} G^{v}(\varphi)\right)^{(1 / v)}+\left(\mathscr{T}_{x^{+}}^{\delta} H^{v}(\varphi)\right)^{(1 / v)} \\
& \quad \leq \frac{1+\Upsilon(\gamma+2)}{(\Upsilon+1)(\gamma+1)}\left(\mathscr{T}_{x^{+}}^{\delta}(G+H)^{v}(\varphi)\right)^{(1 / v),}
\end{aligned}
$$

holds if $0<\gamma \leq(G(z) / H(z)) \leq \Upsilon$, for all $z \in[x, y]$.

Theorem 4 (see [43]). Let $\delta>0, v \geq 1, y>x \geq 0, \Upsilon \geq \gamma>0$, and $G$ and $H$ be two positive functions defined on $[0, \infty)$ such that $\mathscr{T}_{x^{+}}^{\delta} G^{v}(\varphi)<\infty a$ and $\mathscr{T}_{x^{+}}^{\delta} H^{v}(\varphi)<\infty$, for all $\varphi>0$. Then, the inequality

$$
\begin{aligned}
& \left(\mathscr{T}_{x^{+}}^{\delta} G^{v}(\varphi)\right)^{(2 / v)}+\left(\mathscr{T}_{x^{+}}^{\delta} H^{v}(\varphi)\right)^{(2 / v)} \\
& \quad \geq\left(\frac{(1+\gamma)(\Upsilon+1)}{\Upsilon}-2\right)\left(\mathscr{T}_{x^{+}}^{\delta} G^{v}(\varphi)\right)^{(1 / v)}\left(\mathscr{T}_{x^{+}}^{\delta} H^{v}(\varphi)\right)^{(1 / v)},
\end{aligned}
$$

takes place if $0<\gamma \leq(G(z) / H(z)) \leq \Upsilon$, for all $z \in[x, y]$.

Now, we present a new nonlocal fractional operator which is known as the generalized proportional fractional integral operator of a function with respect to another function $\Phi$ introduced by Rashid et al. [9].

Definition 1 (see [9]). Let $\delta>0, \sigma \in(0,1], x, y \in \mathbb{R}$ with $x<y$, and $\Phi$ be an increasing and positive monotone 
function on $(x, y]$ such that $\Phi^{\prime}$ is continuous on $(x, y)$ and $\Phi(0)=0$. Then, the left and right generalized proportional fractional integral operators $\left(\mathscr{T}_{x, \Phi}^{\delta, \sigma} \mathscr{F}\right)(\varphi)$ and $\left(\mathscr{T}_{y, \Phi}^{\delta, \sigma} \mathscr{F}\right)(\varphi)$ of the function $\mathscr{F}$ with respect to the function $\Phi$ of order $\delta>0$ are defined by

$$
\begin{aligned}
& \left(\mathscr{T}_{x, \Phi}^{\delta, \sigma} \mathscr{F}\right)(\varphi)=\frac{1}{\sigma^{\delta} \Gamma(\delta)} \int_{x}^{\varphi} \frac{\exp \left[((\sigma-1) / \sigma)\left(\Phi(\varphi)-\Phi\left(z_{1}\right)\right)\right] \Phi^{\prime}\left(z_{1}\right)}{\left(\Phi(\varphi)-\Phi\left(z_{1}\right)\right)^{1-\delta}} \mathscr{F}\left(z_{1}\right) \mathrm{d} z_{1}, \quad x<\varphi, \\
& \left(\mathscr{T}_{y, \Phi}^{\delta, \sigma} \mathscr{F}\right)(\varphi)=\frac{1}{\sigma^{\delta} \Gamma(\delta)} \int_{\varphi}^{y} \frac{\exp \left[((\sigma-1) / \sigma)\left(\Phi\left(z_{1}\right)-\Phi(\varphi)\right)\right] \Phi^{\prime}\left(z_{1}\right)}{\left(\Phi\left(z_{1}\right)-\Phi(\varphi)\right)^{1-\delta}} \mathscr{F}\left(z_{1}\right) \mathrm{d} z_{1}, \quad \varphi<y,
\end{aligned}
$$

respectively, where $\Gamma(x)=\int_{0}^{\infty} t^{x-1} e^{-t} \mathrm{~d} t$ is the Gamma function [50-52].

Remark 1. Many fractional integral operators are the special cases of (5) and (6). For example,
(1) Let $\Phi(\varphi)=\varphi$. Then, (5) and (6) lead to the left and right generalized proportional fractional integral operators proposed by Jarad et al. [53] as follows:

$$
\begin{aligned}
& \left(\mathscr{T}_{x}^{\delta, \sigma} \mathscr{F}\right)(\varphi)=\frac{1}{\sigma^{\delta} \Gamma(\delta)} \int_{x}^{\varphi} \frac{\exp \left[((\sigma-1) / \sigma)\left(\varphi-z_{1}\right)\right]}{\left(\varphi-z_{1}\right)^{1-\delta}} \mathscr{F}\left(z_{1}\right) \mathrm{d} z_{1}, \quad x<\varphi, \\
& \left(\mathscr{T}_{y}^{\delta, \sigma} \mathscr{F}\right)(\varphi)=\frac{1}{\sigma^{\delta} \Gamma(\delta)} \int_{\varphi}^{y} \frac{\exp \left[((\sigma-1) / \sigma)\left(z_{1}-\varphi\right)\right]}{\left(z_{1}-\varphi\right)^{1-\delta}} \mathscr{F}\left(z_{1}\right) \mathrm{d} z_{1}, \quad \varphi<y .
\end{aligned}
$$

(2) If $\sigma=1$, then (5) and (6) reduce to the left and right generalized Riemann-Liouville fractional integral operators introduced by Kilbas et al. [8] as follows:

$$
\begin{aligned}
& \left(\mathscr{T}_{x, \Phi}^{\delta} \mathscr{F}\right)(\varphi)=\frac{1}{\Gamma(\delta)} \int_{x}^{\varphi} \frac{\Phi^{\prime}\left(z_{1}\right) \mathscr{F}\left(z_{1}\right)}{\left(\Phi(\varphi)-\Phi\left(z_{1}\right)\right)^{1-\delta}} \mathrm{d} z_{1}, \quad x<\varphi \\
& \left(\mathscr{T}_{y, \Phi}^{\delta} \mathscr{F}\right)(\varphi)=\frac{1}{\Gamma(\delta)} \int_{\varphi}^{y} \frac{\mathscr{F}\left(z_{1}\right) \Phi^{\prime}\left(z_{1}\right)}{\left(\Phi\left(z_{1}\right)-\Phi(\varphi)\right)^{1-\delta}} \mathrm{d} z_{1}, \quad \varphi<y .
\end{aligned}
$$

(3) Let $\Phi(\varphi)=\ln \varphi$. Then, (5) and (6) become the left and right generalized proportional Hadamard fractional integral operators [54]:

$$
\begin{aligned}
\left(\mathscr{T}_{x}^{\delta, \sigma} \mathscr{F}\right)(\varphi) & =\frac{1}{\sigma^{\delta} \Gamma(\delta)} \int_{x}^{\varphi} \frac{\exp \left[((\sigma-1) / \sigma)\left(\ln \left(\varphi / z_{1}\right)\right)\right]}{\left(\ln \left(\varphi / z_{1}\right)\right)^{1-\delta}} \frac{\mathscr{F}\left(z_{1}\right)}{z_{1}} \mathrm{~d} z_{1}, \quad x<\varphi, \\
\left(\mathscr{T}_{y}^{\delta, \sigma}\right)(\varphi) & =\frac{1}{\sigma^{\delta} \Gamma(\delta)} \int_{\varphi}^{y} \frac{\exp \left[((\sigma-1) / \sigma)\left(\ln \left(z_{1} / \varphi\right)\right)\right]}{\left(\ln \left(z_{1} / \varphi\right)\right)^{1-\delta}} \frac{\mathscr{F}\left(z_{1}\right)}{z_{1}} \mathrm{~d} z_{1}, \quad \varphi<y .
\end{aligned}
$$


(4) If $\Phi(\varphi)=\ln \varphi$ and $\sigma=1$. Then, (5) and (6) lead to the left and right Hadamard fractional integral operators [8]:

$\mathscr{T}_{x}^{\delta} \mathscr{F}(\varphi)=\frac{1}{\Gamma(\delta)} \int_{x}^{\varphi} \frac{\mathscr{F}\left(z_{1}\right)}{z_{1}\left(\ln \left(\varphi / z_{1}\right)\right)^{1-\delta}} \mathrm{d} z_{1}, \quad x<\varphi$,

$\mathscr{T}_{y}^{\delta} \mathscr{F}(\varphi)=\frac{1}{\Gamma(\delta)} \int_{\varphi}^{y} \frac{\mathscr{F}\left(z_{1}\right)}{z_{1}\left(\ln \left(z_{1} / \varphi\right)\right)^{1-\delta}} \mathrm{d} z_{1}, \quad \varphi<y$.

(5) Let $\Phi(\varphi)=\varphi$ and $\sigma=1$. Then, (5) and (6) become the left and right Riemann-Liouville fractional integral operators:

$$
\begin{aligned}
& \mathscr{T}_{x}^{\delta} \mathscr{F}(\varphi)=\frac{1}{\Gamma(\delta)} \int_{x}^{\varphi} \frac{\mathscr{F}\left(z_{1}\right)}{\left(\varphi-z_{1}\right)^{1-\delta}} \mathrm{d} z_{1}, \quad x<\varphi, \\
& \mathscr{T}_{y}^{\delta} \mathscr{F}(\varphi)=\frac{1}{\Gamma(\delta)} \int_{\varphi}^{y} \frac{\mathscr{F}\left(z_{1}\right)}{\left(z_{1}-\varphi\right)^{1-\delta}} \mathrm{d} z_{1}, \quad \varphi<y .
\end{aligned}
$$

\section{Reverse Minkowski Inequalities via Generalized Proportional Fractional Integral Operator with respect to Another Function}

This segment will consist of several generalizations by using generalized nonlocal proportional fractional integral operator with respect to another function $\Phi$ to derive reverse Minkowski integral inequalities.

Theorem 5. Let $\sigma \in(0,1], \delta>0, v \geq 1, \Upsilon \geq \gamma>0$, $G$ and $H$ be two positive functions defined on $[0, \infty)$ such that $\mathscr{T}_{x, \Phi}^{\delta, \sigma} G^{v}(\varphi)<\infty$ and $\mathscr{T}_{x, \Phi}^{\delta, \sigma} H^{v}(\varphi)<\infty$, for all $\varphi>0$, and $\Phi$ be an increasing and positive function defined on $[0, \infty)$ such that $\Phi^{\prime}$ is continuous on $[0, \infty)$ and $\Phi(0)=0$. Then,

$$
\begin{aligned}
& \left(\mathscr{T}_{x, \Phi}^{\delta, \sigma} G^{v}(\varphi)\right)^{(1 / v)}+\left(\mathscr{T}_{x, \Phi}^{\delta, \sigma} H^{v}(\varphi)\right)^{(1 / v)} \\
& \leq \frac{(1+\Upsilon)(\gamma+2)}{(\gamma+1)(\Upsilon+1)}\left(\mathscr{T}_{x, \Phi}^{\delta, \sigma}(G+H)^{v}(\varphi)\right)^{(1 / v)},
\end{aligned}
$$

if $0<\gamma \leq(G(z) / H(z)) \leq \Upsilon$, for all $z \in[x, \varphi] \subseteq[0, \infty)$.

Proof. It follows from $(G(z) / H(z)) \leq \Upsilon$ for $z_{1} \in[x, \varphi]$ that

$$
(\Upsilon+1)^{v} G^{v}\left(z_{1}\right) \leq \Upsilon^{v}(G+H)^{v}\left(z_{1}\right) .
$$

Multiplying both sides of (13) by

$$
\frac{1}{\sigma^{\delta} \Gamma(\delta)} \frac{\exp \left[((\sigma-1) / \sigma)\left(\Phi(\varphi)-\Phi\left(z_{1}\right)\right)\right] \Phi^{\prime}\left(z_{1}\right)}{\left(\Phi(\varphi)-\Phi\left(z_{1}\right)\right)^{1-\delta}}
$$

and integrating with respect to $z_{1}$ on $(x, \varphi)$, we obtain

$$
\begin{aligned}
& \frac{(\Upsilon+1)^{v}}{\sigma^{\delta} \Gamma(\delta)} \int_{x}^{\varphi} \frac{\exp \left[((\sigma-1) / \sigma)\left(\Phi(\varphi)-\Phi\left(z_{1}\right)\right)\right] \Phi^{\prime}\left(z_{1}\right)}{\left(\Phi(\varphi)-\Phi\left(z_{1}\right)\right)^{1-\delta}} G^{v}\left(z_{1}\right) \mathrm{d} z_{1} \\
& \quad \leq \frac{\Upsilon^{v}}{\sigma^{\delta} \Gamma(\delta)} \int_{x}^{\varphi} \frac{\exp \left[((\sigma-1) / \sigma)\left(\Phi(\varphi)-\Phi\left(z_{1}\right)\right)\right] \Phi^{\prime}\left(z_{1}\right)}{\left(\Phi(\varphi)-\Phi\left(z_{1}\right)\right)^{1-\delta}}(G+H)^{v}\left(z_{1}\right) \mathrm{d} z_{1}
\end{aligned}
$$

which can be written as

$$
\mathscr{T}_{x, \Phi}^{\delta, \sigma} G^{v}(\varphi) \leq \frac{\Upsilon^{v}}{(\Upsilon+1)^{v}} \mathscr{T}_{x, \Phi}^{\delta, \sigma}(G+H)^{v}(\varphi),
$$

that is,

$$
\left(\mathscr{T}_{x, \Phi}^{\delta, \sigma} G^{v}(\varphi)\right)^{(1 / v)} \leq \frac{\Upsilon}{(\Upsilon+1)}\left(\mathscr{T}_{x, \Phi}^{\delta, \sigma}(G+H)^{v}(\varphi)\right)^{(1 / v)}
$$

On the contrary, from $\gamma H\left(z_{1}\right) \leq G\left(z_{1}\right)$, one has

$$
\left(1+\frac{1}{\gamma}\right) H\left(z_{1}\right) \leq \frac{1}{\gamma}\left(G\left(z_{1}\right)+H\left(z_{1}\right)\right),
$$

which leads to

$$
\left(1+\frac{1}{\gamma}\right)^{v} H^{v}\left(z_{1}\right) \leq\left(\frac{1}{\gamma}\right)^{v}\left(G\left(z_{1}\right)+H\left(z_{1}\right)\right)^{v} .
$$

Multiplying both sides of (19) by

$$
\frac{1}{\sigma^{\delta} \Gamma(\delta)} \frac{\exp \left[((\sigma-1) / \sigma)\left(\Phi(\varphi)-\Phi\left(z_{1}\right)\right)\right] \Phi^{\prime}\left(z_{1}\right)}{\left(\Phi(\varphi)-\Phi\left(z_{1}\right)\right)^{1-\delta}}
$$

and integrating with respect to $z_{1}$ on $(x, \varphi)$, we obtain

$$
\left(\mathscr{T}_{x, \Phi}^{\delta, \sigma} H^{v}(\varphi)\right)^{(1 / v)} \leq \frac{1}{(\gamma+1)}\left(\mathscr{T}_{x, \Phi}^{\delta, \sigma}(G+H)^{v}(\varphi)\right)^{(1 / v)} .
$$

Adding inequalities (17) and (21) yields the desired inequality (12).

Remark 2. If $\sigma=1$, then Theorem 5 leads to Theorem 3.1 in [47]. If $\Phi\left(z_{1}\right)=z_{1}$ and $\sigma=1$, then Theorem 5 reduces to inequality (3). If $\Phi\left(z_{1}\right)=z_{1}$ and $\delta=\sigma=1$, then Theorem 5 becomes inequality (1). 
Theorem 6. Let $\sigma \in(0,1], \delta>0, v \geq 1, \Upsilon \geq \gamma>0, G$ and $H$ be two positive functions defined on $[0, \infty)$ such that $\mathscr{T}_{x, \Phi}^{\delta, \sigma} G^{v}(\varphi)<\infty$ and $\mathscr{T}_{x, \Phi}^{\delta, \sigma} H^{v}(\varphi)<\infty$, for all $\varphi>0$, and let $\Phi$ be an increasing and positive function defined on $[0, \infty)$ such that $\Phi^{\prime}$ is continuous on $[0, \infty)$ and $\Phi(0)=0$. Then,

$$
\begin{aligned}
& \left(\mathscr{T}_{x, \Phi}^{\delta, \sigma} G^{v}(\varphi)\right)^{2 / v}+\left(\mathscr{T}_{x, \Phi}^{\delta, \sigma} H^{v}(\varphi)\right)^{2 / v} \\
& \quad \geq\left(\frac{(\Upsilon+1)(\gamma+1)}{\Upsilon}-2\right)\left(\mathscr{T}_{x, \Phi}^{\delta, \sigma} G^{v}(\varphi)\right)^{1 / v}\left(\mathscr{T}_{x, \Phi}^{\delta, \sigma} H^{v}(\varphi)\right)^{1 / v},
\end{aligned}
$$

if $0<\gamma \leq(G(z) / H(z)) \leq \Upsilon$, for all $z \in[x, \varphi] \subseteq[0, \infty)$.

Proof. Carrying out product between (17) and (21) yields

$$
\begin{aligned}
& \left(\frac{(\Upsilon+1)(\gamma+1)}{\Upsilon}\right)\left(\mathscr{T}_{x, \Phi}^{\delta, \sigma} G^{v}(\varphi)\right)^{1 / v}\left(\mathscr{T}_{x, \Phi}^{\delta, \sigma} H^{v}(\varphi)\right)^{1 / v} \\
& \quad \leq\left[\left(\mathscr{T}_{x, \Phi}^{\delta, \sigma}(G(\varphi)+H(\varphi))^{v}\right)^{1 / v}\right]^{2} .
\end{aligned}
$$

Applying the Minkowski inequality to the right-hand side of (23), we obtain

$$
\begin{aligned}
{\left[\left(\mathscr{T}_{x, \Phi}^{\delta, \sigma}(G(\varphi)+H(\varphi))^{v}\right)^{1 / v}\right]^{2} } \\
\leq\left[\left(\mathscr{T}_{x, \Phi}^{\delta, \sigma} G^{v}(\varphi)\right)^{1 / v}+\left(\mathscr{T}_{x, \Phi}^{\delta, \sigma} H(\varphi)\right)^{1 / v}\right]^{2} \\
\leq\left(\mathscr{T}_{x, \Phi}^{\delta, \sigma} G^{v}(\varphi)\right)^{2 / v}+\left(\mathscr{T}_{x, \Phi}^{\delta, \sigma} H(\varphi)\right)^{2 / v} \\
\quad+2\left(\mathscr{T}_{x, \Phi}^{\delta, \sigma} G^{v}(\varphi)\right)^{1 / v}\left(\mathscr{T}_{x, \Phi}^{\delta, \sigma} H(\varphi)\right)^{1 / v} .
\end{aligned}
$$

It follows from (23) and (24) that

$$
\begin{aligned}
& \left(\frac{(\Upsilon+1)(\gamma+1)}{\Upsilon}-2\right)\left(\mathscr{T}_{x, \Phi}^{\delta, \sigma} G^{v}(\varphi)\right)^{1 / v}\left(\mathscr{T}_{x, \Phi}^{\delta, \sigma} H^{v}(\varphi)\right)^{1 / v} \\
& \leq\left(\left(\mathscr{T}_{x, \Phi}^{\delta, \sigma} G^{v}(\varphi)\right)^{1 / v}+\left(\mathscr{T}_{x, \Phi}^{\delta, \sigma} H^{v}(\varphi)\right)^{1 / v}\right)^{2} .
\end{aligned}
$$

Inequality (25) leads to the conclusion that

$$
\begin{aligned}
& \left(\mathscr{T}_{x, \Phi}^{\delta, \sigma} G^{v}(\varphi)\right)^{2 / v}+\left(\mathscr{T}_{x, \Phi}^{\delta, \sigma} H^{v}(\varphi)\right)^{2 / v} \\
& \quad \geq\left(\frac{(\Upsilon+1)(\gamma+1)}{\Upsilon}-2\right)\left(\mathscr{T}_{x, \Phi}^{\delta, \sigma} G^{v}(\varphi)\right)^{1 / v}\left(\mathscr{T}_{x, \Phi}^{\delta, \sigma} H^{v}(\varphi)\right)^{1 / v},
\end{aligned}
$$

which complete the proof of Theorem 6 .

Remark 3. If $\sigma=1$, then Theorem 6 leads to Theorem 3.2 of [47]; if $\Phi\left(z_{1}\right)=z_{1}$ and $\sigma=1$, then Theorem 6 reduces to inequality (4); if $\Phi\left(z_{1}\right)=z_{1}$ and $\delta=\sigma=1$, then Theorem 6 becomes inequality (2).

\section{Some Estimates for the Generalized Proportional Fractional Integral Operator with Respect to Another Function}

This section is consisted to establishing several associated variants concerning to the generalized proportional fractional integral operator with respect to another function $\Phi$.

Theorem 7. Let $\sigma \in(0,1], \delta>0, \Upsilon \geq \gamma>0, v_{1}, v_{2}>1$ with $\left(1 / v_{1}\right)+\left(1 / v_{2}\right)=1, G$ and $H$ be two positive functions defined on $[0, \infty)$ such that $\mathscr{T}_{x, \Phi}^{\delta, \sigma} G(\varphi)<\infty$ and $\mathscr{T}_{x, \Phi}^{\delta, \sigma} H(\varphi)<\infty$ for $\varphi>0$, and $\Phi$ be an increasing and positive function defined on $[0, \infty)$ such that $\Phi^{\prime}$ is continuous on $[0, \infty)$ and $\Phi(0)=0$. Then, one has

$$
\begin{gathered}
\left(\mathscr{T}_{x, \Phi}^{\delta, \sigma} G(\varphi)\right)^{1 / v_{1}}\left(\mathscr{T}_{x, \Phi}^{\delta, \sigma} H(\varphi)\right)^{1 / v_{2}} \\
\leq\left(\frac{\Upsilon}{\gamma}\right)^{1 / v_{1} v_{2}}\left(\mathscr{T}_{x, \Phi}^{\delta, \sigma} G^{1 / v_{1}}(\varphi) H^{1 / v_{2}}(\varphi)\right), \\
\text { if } 0<\gamma \leq(G(z) / H(z)) \leq \Upsilon \text {, for all } z_{1} \in[x, \varphi] \subseteq[0, \infty) .
\end{gathered}
$$

Proof. It follows from $\left(G\left(z_{1}\right) / H\left(z_{1}\right)\right) \leq \Upsilon$ for $z_{1} \in[x, \varphi]$ that

$$
\left(H\left(z_{1}\right)\right)^{1 / v_{2}} \geq \Upsilon^{-\left(1 / v_{2}\right)}\left(G\left(z_{1}\right)\right)^{1 / v_{2}}
$$

Multiplying both sides of (28) by $G^{1 / v_{1}}\left(z_{1}\right)$ leads to

$$
\left(G^{1 / v_{1}}\left(z_{1}\right)\right)\left(H^{1 / v_{2}}\left(z_{1}\right)\right) \geq \Upsilon^{-\left(1 / v_{2}\right)}\left(G\left(z_{1}\right)\right) .
$$

Multiplying on both sides of (28) by

$$
\frac{1}{\sigma^{\delta} \Gamma(\delta)} \frac{\exp \left[((\sigma-1) / \sigma)\left(\Phi(\varphi)-\Phi\left(z_{1}\right)\right)\right] \Phi^{\prime}\left(z_{1}\right)}{\left(\Phi(\varphi)-\Phi\left(z_{1}\right)\right)^{1-\delta}}
$$

and integrating with respect to $z_{1}$ on $(x, \varphi)$, we obtain 


$$
\begin{aligned}
& \frac{\Upsilon^{-\left(1 / v_{2}\right)}}{\sigma^{\delta} \Gamma(\delta)} \int_{x}^{\varphi} \frac{\exp \left[((\sigma-1) / \sigma)\left(\Phi(\varphi)-\Phi\left(z_{1}\right)\right)\right] \Phi^{\prime}\left(z_{1}\right)}{\left(\Phi(\varphi)-\Phi\left(z_{1}\right)\right)^{1-\delta}}\left(G\left(z_{1}\right)\right) \mathrm{d} z_{1} \\
& \quad \leq \frac{1}{\sigma^{\delta} \Gamma(\delta)} \int_{x}^{\varphi} \frac{\exp \left[((\sigma-1) / \sigma)\left(\Phi(\varphi)-\Phi\left(z_{1}\right)\right)\right] \Phi^{\prime}\left(z_{1}\right)}{\left(\Phi(\varphi)-\Phi\left(z_{1}\right)\right)^{1-\delta}}\left(G^{1 / v_{1}}\left(z_{1}\right)\right)\left(H^{1 / v_{2}}\left(z_{1}\right)\right) \mathrm{d} z_{1} .
\end{aligned}
$$

Inequality (31) can be written as

$\Upsilon^{-\left(1 / v_{1} v_{2}\right)}\left[\mathscr{T}_{x, \Phi}^{\delta, \sigma} G(\varphi)\right]^{1 / v_{1}} \leq\left[\mathscr{T}_{x, \Phi}^{\delta, \sigma}\left([G(\varphi)]^{1 / v_{1}}[H(\varphi)]^{1 / v_{2}}\right)\right]^{1 / v_{1}}$.

On the contrary, $\gamma H\left(z_{1}\right) \leq G\left(z_{1}\right)$ leads to

$$
\gamma^{1 / v_{1}} H^{1 / v_{1}}\left(z_{1}\right) \leq G^{1 / v_{1}}\left(z_{1}\right) \text {. }
$$

Multiplying on both sides of (34) by

$$
\frac{1}{\sigma^{\delta} \Gamma(\delta)} \frac{\exp \left[((\sigma-1) / \sigma)\left(\Phi(\varphi)-\Phi\left(z_{1}\right)\right)\right] \Phi^{\prime}\left(z_{1}\right)}{\left(\Phi(\varphi)-\Phi\left(z_{1}\right)\right)^{1-\delta}}
$$

Multiplying on both sides of (33) by $H^{1 / v_{2}}\left(z_{1}\right)$ and using the identity $v_{1}^{-1}+v_{2}^{-1}=1$, we have

$$
\begin{aligned}
& \frac{\gamma^{\left(1 / v_{1}\right)}}{\sigma^{\delta} \Gamma(\delta)} \int_{x}^{\varphi} \frac{\exp \left[((\sigma-1) / \sigma)\left(\Phi(\varphi)-\Phi\left(z_{1}\right)\right)\right] \Phi^{\prime}\left(z_{1}\right)}{\left(\Phi(\varphi)-\Phi\left(z_{1}\right)\right)^{1-\delta}} H\left(z_{1}\right) \mathrm{d} z_{1} \\
& \quad \leq \frac{1}{\sigma^{\delta} \Gamma(\delta)} \int_{x}^{\varphi} \frac{\exp \left[((\sigma-1) / \sigma)\left(\Phi(\varphi)-\Phi\left(z_{1}\right)\right)\right] \Phi^{\prime}\left(z_{1}\right)}{\left(\Phi(\varphi)-\Phi\left(z_{1}\right)\right)^{1-\delta}} H^{1 / v_{1}}\left(z_{1}\right) G^{1 / v_{2}}\left(z_{1}\right) \mathrm{d} z_{1}
\end{aligned}
$$

Inequality (36) leads to

$$
\gamma^{1 / v_{1} v_{2}}\left(\mathscr{T}_{x, \Phi}^{\delta, \sigma} H(\varphi)\right)^{1 / v_{2}} \leq\left(\mathscr{T}_{x, \Phi}^{\delta, \sigma} H^{1 / v_{1}}(\varphi) G^{\left(1 / v_{2}\right)}(\varphi)\right)^{1 / v_{2}}
$$

From (32) and (37), together with $v_{1}^{-1}+v_{2}^{-1}=1$, we clearly see that 


$$
\begin{aligned}
& \frac{(\Upsilon+1)^{v_{1}}}{\sigma^{\delta} \Gamma(\delta)} \int_{x}^{\varphi} \frac{\exp \left[((\sigma-1) / \sigma) \Phi\left((\varphi)-\Phi\left(z_{1}\right) \Phi^{\prime}\left(z_{1}\right)\right)\right] \Phi^{\prime}\left(z_{1}\right)}{\left(\Phi(\varphi)-\Phi\left(z_{1}\right)\right)^{1-\delta}} G^{v_{1}}\left(z_{1}\right) \mathrm{d} z_{1} \\
& \quad \leq \frac{\Upsilon^{v_{1}}}{\sigma^{\delta} \Gamma(\delta)} \int_{x}^{\varphi} \frac{\exp \left[((\sigma-1) / \sigma)\left(\Phi(\varphi)-\Phi\left(z_{1}\right)\right)\right] \Phi^{\prime}\left(z_{1}\right)}{\left(\Phi(\varphi)-\Phi\left(z_{1}\right)\right)^{1-\delta}}(G+H)^{v_{1}}\left(z_{1}\right) \mathrm{d} z_{1} .
\end{aligned}
$$

Inequality (42) can be written as

$$
\mathscr{T}_{x, \Phi}^{\delta, \sigma} G^{v_{1}}(\varphi) \leq \frac{\Upsilon^{v_{1}}}{(\Upsilon+1)^{v_{1}}} \mathscr{T}_{x, \Phi}^{\delta, \sigma}(G+H)^{v_{1}}(\varphi) .
$$

On the contrary, it follows from $\left(G\left(z_{1}\right) / H\left(z_{1}\right)\right)>\Upsilon$ that

$$
(\gamma+1)^{v_{2}} H^{v_{2}}\left(z_{1}\right) \leq(G+H)^{v_{2}}\left(z_{1}\right) .
$$

Multiplying on both sides of (44) by

$$
\frac{1}{\sigma^{\delta} \Gamma(\delta)} \frac{\exp \left[((\sigma-1) / \sigma)\left(\Phi(\varphi)-\Phi\left(z_{1}\right)\right)\right] \Phi \prime\left(z_{1}\right)}{\left(\Phi(\varphi)-\Phi\left(z_{1}\right)\right)^{1-\delta}}
$$

and integrating with respect to $z_{1}$ on $(x, \varphi)$, we obtain

$$
\mathscr{T}_{x, \Phi}^{\delta, \sigma} H^{v_{2}}(\varphi) \leq \frac{1}{(\gamma+1)^{v_{2}}} \mathscr{T}_{x, \Phi}^{\delta, \sigma}(G+H)^{v_{2}}(\varphi) .
$$

The well-known Young's inequality states that

$$
\frac{G^{v_{1}}\left(z_{1}\right)}{v_{1}}+\frac{H^{v_{2}}\left(z_{1}\right)}{v_{2}} \geq G\left(z_{1}\right) H\left(z_{1}\right)
$$

Multiplying both sides of (47) with

$$
\frac{1}{\sigma^{\delta} \Gamma(\delta)} \frac{\exp \left[((\sigma-1) / \sigma)\left(\Phi(\varphi)-\Phi\left(z_{1}\right)\right)\right] \Phi^{\prime}\left(z_{1}\right)}{\left(\Phi(\varphi)-\Phi\left(z_{1}\right)\right)^{1-\delta}}
$$

and integrating with respect to $z_{1}$ on $(x, \varphi)$ give

$$
\frac{1}{v_{1}}\left(\mathscr{T}_{x, \Phi}^{\delta, \sigma} G^{v_{1}}(\varphi)\right)+\frac{1}{v_{2}}\left(\mathscr{T}_{x, \Phi}^{\delta, \sigma} H^{v_{2}}(\varphi)\right) \geq \mathscr{T}_{x, \Phi}^{\delta, \sigma}(G(\varphi) H(\varphi)) .
$$

From (43), (46), and (49), we clearly see that

$$
\mathscr{T}_{x, \Phi}^{\delta, \sigma}(G(\varphi) H(\varphi)) \leq \frac{1}{v_{1}}\left(\mathscr{T}_{x, \Phi}^{\delta, \sigma} G^{v_{1}}(\varphi)\right)+\frac{1}{v_{2}}\left(\mathscr{T}_{x, \Phi}^{\delta, \sigma} H^{v_{2}}(\varphi)\right) \leq \frac{\Upsilon^{v_{1}}}{v_{1}(\Upsilon+1)^{v_{1}}} \mathscr{T}_{x, \Phi}^{\delta, \sigma}(G+H)^{v_{1}}(\varphi)+\frac{1}{v_{2}(\gamma+1)^{v_{2}}} \mathscr{T}_{x, \Phi}^{\delta, \sigma}(G+H)^{v_{2}}(\varphi)
$$

Making use of the inequality $\left(a_{1}+a_{2}\right)^{q} \leq 2^{q-1}\left(a_{1}^{q}+a_{2}^{q}\right)$, for $a_{1}, a_{2}>0$ and $q>1$, we can obtain

$$
\begin{aligned}
& \mathscr{T}_{x, \Phi}^{\delta, \sigma}(G+H)^{v_{1}}(\varphi) \leq 2^{v_{1}-1} \mathscr{T}_{x, \Phi}^{\delta, \sigma}\left(G^{v_{1}}+H^{v_{1}}\right)(\varphi), \\
& \mathscr{T}_{x, \Phi}^{\delta, \sigma}(G+H)^{v_{2}}(\varphi) \leq 2^{v_{2}-1} \mathscr{T}_{x, \Phi}^{\delta, \sigma}\left(G^{v_{2}}+H^{v_{2}}\right)(\varphi) .
\end{aligned}
$$

Therefore, inequality (39) follows easily from inequalities (50)-(52).

Theorem 9. Let $\sigma \in(0,1], \delta>0, \Upsilon \geq \gamma>0, v \geq 1, G$ and $H$ be two positive functions defined on $[0, \infty)$ such that $\mathscr{T}_{x, \Phi}^{\delta, \sigma} G(\varphi)<\infty$ and $\mathscr{T}_{x, \Phi}^{\delta, \sigma} H(\varphi)<\infty$ for $\varphi>0$, and $\Phi$ be an increasing and positive function defined on $[0, \infty)$ such that $\Phi^{\prime}$ is continuous on $[0, \infty)$ and $\Phi(0)=0$. Then, one has

$$
\begin{aligned}
\frac{\Upsilon+1}{\Upsilon-\omega}\left(\mathscr{T}_{x, \Phi}^{\delta, \sigma}[G(\varphi)-\omega H(\varphi)]^{v}\right)^{1 / v} \leq & \left(\mathscr{T}_{x, \Phi}^{\delta, \sigma} G^{v}(\varphi)\right)^{(1 / v)} \\
& +\left(\mathscr{T}_{x, \Phi}^{\delta, \sigma} H^{v}(\varphi)\right)^{1 / v} \\
\leq & \frac{\gamma+1}{\gamma-\omega}\left(\mathscr{T}_{x, \Phi}^{\delta, \sigma}[G(\varphi)-\omega H(\varphi)]^{v}\right)^{1 / v},
\end{aligned}
$$

if $0<\omega<\gamma \leq\left(G\left(z_{1}\right) / H\left(z_{1}\right)\right) \leq \Upsilon$, for all $z_{1} \in[x, \varphi] \subseteq[0, \infty)$.
Proof. It follows from $0<\omega<\gamma \leq\left(G\left(z_{1}\right) / H\left(z_{1}\right)\right) \leq \Upsilon$ that $\gamma \omega \leq \Upsilon \omega$

$$
\gamma \omega+\gamma \leq \gamma \omega+\Upsilon \leq \Upsilon \omega+\Upsilon,
$$$$
(\Upsilon+1)(\gamma-\omega) \leq(\gamma+1)(\Upsilon-\omega) \text {, }
$$$$
\frac{\Upsilon+1}{\Upsilon-\omega} \leq \frac{\gamma+1}{\gamma-\omega}
$$$$
\gamma-\omega \leq \frac{G\left(z_{1}\right)-\omega H\left(z_{1}\right)}{H\left(z_{1}\right)} \leq \Upsilon-\omega
$$$$
\frac{\left(G\left(z_{1}\right)-\omega H\left(z_{1}\right)\right)^{v}}{(\Upsilon-\omega)^{v}} \leq H^{v}\left(z_{1}\right) \leq \frac{\left(G\left(z_{1}\right)-\omega H\left(z_{1}\right)\right)^{v}}{(\gamma-\omega)^{v}}
$$$$
\frac{1}{\Upsilon} \leq \frac{H\left(z_{1}\right)}{G\left(z_{1}\right)} \leq \frac{1}{\gamma}
$$$$
\frac{\gamma-\omega}{\gamma \omega} \leq \frac{G\left(z_{1}\right)-\omega H\left(z_{1}\right)}{\omega G\left(z_{1}\right)} \leq \frac{\Upsilon-\omega}{\omega \Upsilon} \text {, }
$$

$$
\left.\left(\frac{\Upsilon}{\Upsilon-\omega}\right)^{v} G\left(z_{1}\right)-\omega H\left(z_{1}\right)^{v} \leq G\left(z_{1}\right)\right)^{v} \leq\left(\frac{\gamma}{\gamma-\omega}\right)^{v}\left(G\left(z_{1}\right)-\omega H\left(z_{1}\right)\right)^{v}
$$


Multiplying both sides of (54) by

$$
\frac{1}{\sigma^{\delta} \Gamma(\delta)} \frac{\exp \left[(((\sigma-1) / \sigma))\left(\Phi(\varphi)-\Phi\left(z_{1}\right)\right)\right] \Phi^{\prime}\left(z_{1}\right)}{\left(\Phi(\varphi)-\Phi\left(z_{1}\right)\right)^{1-\delta}}
$$

$$
\begin{aligned}
& \frac{1}{(\Upsilon-\omega)^{v} \sigma^{\delta} \Gamma(\delta)} \int_{x}^{\varphi} \frac{\exp \left[((\sigma-1) / \sigma)\left(\Phi(\varphi)-\Phi\left(z_{1}\right)\right)\right] \Phi^{\prime}\left(z_{1}\right)}{\left(\Phi(\varphi)-\Phi\left(z_{1}\right)\right)^{1-\delta}}\left(G\left(z_{1}\right)-\omega H\left(z_{1}\right)\right)^{v} \mathrm{~d} z_{1} \\
& \leq \frac{1}{\sigma^{\delta} \Gamma(\delta)} \int_{x}^{\varphi} \int_{x} \frac{\exp \left[((\sigma-1) / \sigma)\left(\Phi(\varphi)-\Phi\left(z_{1}\right)\right)\right] \Phi^{\prime}\left(z_{1}\right)}{\left(\Phi(\varphi)-\Phi\left(z_{1}\right)\right)^{1-\delta}} H^{v}\left(z_{1}\right) \mathrm{d} z_{1} \\
& \leq \frac{1}{(\gamma-\omega)^{v} \sigma^{\delta} \Gamma(\delta)} \int_{x}^{\varphi} \frac{\exp \left[((\sigma-1) / \sigma)\left(\Phi(\varphi)-\Phi\left(z_{1}\right)\right)\right] \Phi^{\prime}\left(z_{1}\right)}{\left(\Phi(\varphi)-\Phi\left(z_{1}\right)\right)^{1-\delta}}\left(G\left(z_{1}\right)-\omega H\left(z_{1}\right)\right)^{v} \mathrm{~d} z_{1} .
\end{aligned}
$$

Inequality (57) can be rewritten as

$$
\begin{aligned}
\frac{1}{\gamma-\omega}\left(\mathscr{T}_{x, \Phi}^{\delta, \sigma}(G(\varphi)-\omega H(\varphi))^{v}\right)^{1 / v} & \leq\left(\mathscr{T}_{x, \Phi}^{\delta, \sigma} H^{v}(\varphi)\right)^{1 / v} \\
& \leq \frac{1}{\gamma-\omega}\left(\mathscr{T}_{x, \Phi}^{\delta, \sigma}(G(\varphi)-\omega H(\varphi))^{v}\right)^{1 / v} .
\end{aligned}
$$

Again, multiplying both sides of (55) with

$$
\frac{1}{\sigma^{\delta} \Gamma(\delta)} \frac{\exp \left[((\sigma-1) / \sigma)\left(\Phi(\varphi)-\Phi\left(z_{1}\right)\right)\right] \Phi^{\prime}\left(z_{1}\right)}{\left(\Phi(\varphi)-\Phi\left(z_{1}\right)\right)^{1-\delta}}
$$

$$
\begin{aligned}
& \frac{\Upsilon}{\Upsilon-\omega}\left(\mathscr{T}_{x, \Phi}^{\delta, \sigma}(G(\varphi)-\omega H(\varphi))^{v}\right)^{1 / v} \leq\left(\mathscr{T}_{x, \Phi}^{\delta, \sigma} G^{v}(\varphi)\right)^{1 / v} \\
& \leq \frac{\gamma}{\gamma-\omega}\left(\mathscr{T}_{x, \Phi}^{\delta, \sigma}(G(\varphi)-\omega H(\varphi))^{v}\right)^{1 / v} .
\end{aligned}
$$
(60)

Therefore, inequality (53) follows from (58) and

Theorem 10. Let $\sigma \in(0,1], \quad \delta>0, \quad v \geq 1, \quad 0 \leq \kappa \leq \mathscr{K}$, $0 \leq \mathscr{M} \leq \mathscr{M}, G$ and $H$ be two positive functions defined on

$[0, \infty)$ such that $\mathscr{T}_{x, \Phi}^{\delta, \sigma} G^{v}(\varphi)<\infty$ and $\mathscr{T}_{x, \Phi}^{\delta, \sigma} H^{v}(\varphi)<\infty$ for $\varphi>0$, and $\Phi$ be an increasing and positive function defined on $[0, \infty)$ such that $\Phi^{\prime}$ is continuous on $[0, \infty)$ and $\Phi(0)=0$. Then, the inequality

$$
\left(\mathscr{T}_{x, \Phi}^{\delta, \sigma} G^{v}(\varphi)\right)^{1 / v}+\left(\mathscr{T}_{x, \Phi}^{\delta, \sigma} H^{v}(\varphi)\right)^{1 / v} \leq \frac{\mathscr{K}(\kappa+\mathscr{M})+\mathscr{M}(\mathscr{M}+\mathscr{K})}{(\mathscr{M}+\mathscr{K})(\kappa+\mathscr{M})}\left(\mathscr{T}_{x, \Phi}^{\delta, \sigma}(G(\varphi)+H(\varphi))^{v}(\varphi)\right)^{1 / v}
$$

holds if $\kappa \leq G\left(z_{1}\right) \leq \mathscr{K}$ and $\mathscr{M} \leq H\left(z_{1}\right) \leq \mathscr{M}$, for all $z_{1} \in[x, \varphi]$.

Proof. It follows from the conditions given in Theorem 10 that

$$
\frac{1}{\mathscr{M}} \leq \frac{1}{H\left(z_{1}\right)} \leq \frac{1}{\mathscr{M}}
$$

Inequality (62) and $0 \leq \kappa \leq G\left(z_{1}\right) \leq \mathscr{K}$ lead to the conclusion that

$$
\frac{\kappa}{\mathscr{M}} \leq \frac{G\left(z_{1}\right)}{H\left(z_{1}\right)} \leq \frac{\mathscr{K}}{\mathscr{M}} .
$$

From (63), we clearly see that

$$
\begin{aligned}
& H^{v}\left(z_{1}\right) \leq\left(\frac{\mathscr{M}}{\kappa+\mathscr{M}}\right)^{v}\left(G\left(z_{1}\right)+H\left(z_{1}\right)\right)^{v}, \\
& G^{v}\left(z_{1}\right) \leq\left(\frac{\mathscr{K}}{\mathscr{M}+\mathscr{K}}\right)^{v}\left(G\left(z_{1}\right)+H\left(z_{1}\right)\right)^{v} .
\end{aligned}
$$

Multiplying both sides of (64) and (65) by 


$$
\frac{1}{\sigma^{\delta} \Gamma(\delta)} \frac{\exp \left[((\sigma-1) / \sigma)\left(\Phi(\varphi)-\Phi\left(z_{1}\right)\right)\right] \Phi^{\prime}\left(z_{1}\right)}{\left(\Phi(\varphi)-\Phi\left(z_{1}\right)\right)^{1-\delta}}
$$

and integrating with respect to $z_{1}$ on $(x, \varphi)$, we obtain

$$
\begin{gathered}
\left(\mathscr{T}_{x, \Phi}^{\delta, \sigma} H^{v}(\varphi)\right)^{1 / v} \leq\left(\frac{\mathscr{M}}{\mathcal{K}+\mathscr{M}}\right)\left(\mathscr{T}_{x, \Phi}^{\delta, \sigma}\left(G\left(z_{1}\right)+H\left(z_{1}\right)\right)^{v}\right)^{1 / v}, \\
\left(\mathscr{T}_{x, \Phi}^{\delta, \sigma} G^{v}(\varphi)\right)^{1 / v} \leq\left(\frac{\mathscr{K}}{\mathscr{M}+\mathscr{K}}\right)\left(\mathscr{T}_{x, \Phi}^{\delta, \sigma}\left(G\left(z_{1}\right)+H\left(z_{1}\right)\right)^{v}\right)^{1 / v} .
\end{gathered}
$$
(68).

Therefore, inequality (61) follows from (67) and

Theorem 11. Let $\sigma \in(0,1], \delta>0, v \geq 1,0<\gamma \leq \Upsilon, G$ and $H$ be two positive functions defined on $[0, \infty)$ such that $\mathscr{T}_{x, \Phi}^{\delta, \sigma} G^{v}(\varphi)<\infty$ and $\mathscr{T}_{x, \Phi}^{\delta, \sigma} H^{v}(\varphi)<\infty$ for all $\varphi>0$, and $\Phi$ be an increasing and positive function defined on $[0, \infty)$ such that $\Phi^{\prime}$ is continuous on $[0, \infty)$ and $\Phi(0)=0$. Then, the double inequality

$$
\frac{\left(\mathscr{T}_{x, \Phi}^{\delta, \sigma} G(\varphi) H(\varphi)\right)}{\Upsilon} \leq \frac{\left(\mathscr{T}_{x, \Phi}^{\delta, \sigma}(G+H)^{2}(\varphi)\right)}{(\gamma+1)(\Upsilon+1)} \leq \frac{\left(\mathscr{T}_{x, \Phi}^{\delta, \sigma} G(\varphi) H(\varphi)\right)}{\gamma}
$$

holds if $0<\gamma \leq\left(G\left(z_{1}\right) / H\left(z_{1}\right)\right) \leq \Upsilon$, for all $z_{1} \in[x, \varphi]$.

Proof. It follows from $0<\gamma \leq\left(G\left(z_{1}\right) / H\left(z_{1}\right)\right) \leq \Upsilon$ that

$$
\begin{gathered}
H\left(z_{1}\right)(\gamma+1) \leq H\left(z_{1}\right)+G\left(z_{1}\right) \leq H\left(z_{1}\right)(\Upsilon+1), \\
G\left(z_{1}\right)\left(\frac{\Upsilon+1}{\Upsilon}\right) \leq H\left(z_{1}\right)+G\left(z_{1}\right) \leq G\left(z_{1}\right)\left(\frac{\gamma+1}{\gamma}\right) .
\end{gathered}
$$

Inequality (70) and (71) lead to

$$
\frac{G\left(z_{1}\right) H\left(z_{1}\right)}{\Upsilon} \leq \frac{\left(G\left(z_{1}\right)+H\left(z_{1}\right)\right)^{2}}{(\gamma+1)(\Upsilon+1)} \leq \frac{G\left(z_{1}\right) H\left(z_{1}\right)}{\gamma} .
$$

Multiplying both sides of (72) with

$$
\frac{1}{\sigma^{\delta} \Gamma(\delta)} \frac{\exp \left[((\sigma-1) / \sigma)\left(\Phi(\varphi)-\Phi\left(z_{1}\right)\right)\right] \Phi^{\prime}\left(z_{1}\right)}{\left(\Phi(\varphi)-\Phi\left(z_{1}\right)\right)^{1-\delta}}
$$

and integrating with respect to $z_{1}$ on $(x, \varphi)$, we obtain

$$
\begin{aligned}
& \frac{1}{\Upsilon \sigma^{\delta} \Gamma(\delta)} \int_{x}^{\varphi} \frac{\exp \left[((\sigma-1) / \sigma)\left(\Phi(\varphi)-\Phi\left(z_{1}\right)\right)\right] \Phi^{\prime}\left(z_{1}\right)}{\left(\Phi(\varphi)-\Phi\left(z_{1}\right)\right)^{1-\delta}} G\left(z_{1}\right) H\left(z_{1}\right) \mathrm{d} z_{1} \\
& \quad \leq \frac{1}{(\gamma+1)(\Upsilon+1) \sigma^{\delta} \Gamma(\delta)} \int_{x}^{\varphi} \frac{\exp \left[((\sigma-1) / \sigma)\left(\Phi(\varphi)-\Phi\left(z_{1}\right)\right)\right] \Phi^{\prime}\left(z_{1}\right)}{\left(\Phi(\varphi)-\Phi\left(z_{1}\right)\right)^{1-\delta}}\left(G\left(z_{1}\right)+H\left(z_{1}\right)\right)^{2} \mathrm{~d} z_{1} \\
& \quad \leq \frac{1}{\gamma \sigma^{\delta} \Gamma(\delta)} \int_{x}^{\varphi} \frac{\exp \left[((\sigma-1) / \sigma)\left(\Phi(\varphi)-\Phi\left(z_{1}\right)\right)\right] \Phi^{\prime}\left(z_{1}\right)}{\left(\Phi\left(z_{1}\right)-\Phi\left(z_{1}\right)\right)^{1-\delta}} G\left(z_{1}\right) H\left(z_{1}\right) \mathrm{d} z_{1} .
\end{aligned}
$$

Inequality (74) can be rewritten as

$\frac{\left(\mathscr{T}_{x, \Phi}^{\delta, \sigma} G(\varphi) H(\varphi)\right)}{\Upsilon} \leq \frac{\left(\mathscr{T}_{x, \Phi}^{\delta, \sigma}(G+H)^{2}(\varphi)\right)}{(\gamma+1)(\Upsilon+1)} \leq \frac{\left(\mathscr{T}_{x, \Phi}^{\delta, \sigma} G(\varphi) H(\varphi)\right)}{\gamma}$.
Theorem 12. Let $\sigma \in(0,1], \delta>0, v \geq 1,0<\gamma \leq \Upsilon, G$ and $H$ be two positive functions defined on $[0, \infty)$ such that $\mathscr{T}_{x, \Phi}^{\delta, \sigma} G^{v}(\varphi)<\infty$ and $\mathscr{T}_{x, \Phi}^{\delta, \sigma} H^{v}(\varphi)<\infty$ for all $\varphi>0$, and $\Phi$ be an increasing and positive function defined on $[0, \infty)$ such that $\Phi^{\prime}$ is continuous on $[0, \infty)$ and $\Phi(0)=0$. Then, the inequality

$$
\left(\mathscr{T}_{x, \Phi}^{\delta, \sigma} G^{v}(\varphi)\right)^{1 / v}+\left(\mathscr{T}_{x, \Phi}^{\delta, \sigma} H^{v}(\varphi)\right)^{1 / v} \leq 2\left(\mathscr{T}_{x, \Phi}^{\delta, \sigma} \Delta^{v}(G(\varphi), H(\varphi))\right)^{1 / v}
$$

holds if $\gamma \leq\left(\left(G\left(z_{1}\right)\right) /\left(H\left(z_{1}\right)\right)\right) \leq \Upsilon$ for all $z_{1} \in[x, \varphi]$, where

$$
\Delta(G(\varphi), H(\varphi))=\max \left\{\Upsilon\left[\left(\frac{\Upsilon}{\gamma}+1\right) G\left(z_{1}\right)-\Upsilon H\left(z_{1}\right)\right], \frac{(\gamma+\Upsilon) H\left(z_{1}\right)-G\left(z_{1}\right)}{\gamma}\right\} .
$$

Proof. It follows from $\gamma \leq(G(z) / H(z)) \leq \Upsilon$ that 


$$
\begin{aligned}
& \gamma \leq \Upsilon+\gamma-\frac{G\left(z_{1}\right)}{H\left(z_{1}\right)}, \\
& \Upsilon+\gamma-\frac{G\left(z_{1}\right)}{H\left(z_{1}\right)} \leq \Upsilon .
\end{aligned}
$$

From (78) and (79), we clearly see that

$$
H\left(z_{1}\right)<\frac{(\Upsilon+\gamma) H\left(z_{1}\right)-G\left(z_{1}\right)}{\gamma} \leq \Delta(G(\varphi), H(\varphi)),
$$

where

$$
\Delta(G(\varphi), H(\varphi))=\max \left\{\Upsilon\left[\left(\frac{\Upsilon}{\gamma}+1\right) G\left(z_{1}\right)-\Upsilon H\left(z_{1}\right)\right], \frac{(\gamma+\Upsilon) H\left(z_{1}\right)-G\left(z_{1}\right)}{\gamma}\right\} .
$$
have

Similarly, from $0<(1 / \Upsilon) \leq\left(H\left(z_{1}\right) / G\left(z_{1}\right)\right) \leq(1 / \gamma)$, we

$$
\begin{aligned}
& \frac{1}{\Upsilon} \leq \frac{1}{\Upsilon}+\frac{1}{\gamma}-\frac{H\left(z_{1}\right)}{G\left(z_{1}\right)}, \\
& \frac{1}{\Upsilon}+\frac{1}{\gamma}-\frac{H\left(z_{1}\right)}{G\left(z_{1}\right)} \leq \frac{1}{\gamma} .
\end{aligned}
$$

Inequalities (82) and (83) lead to

$$
\frac{1}{\Upsilon} \leq \frac{((1 / \Upsilon)+(1 / \gamma)) G\left(z_{1}\right)-H\left(z_{1}\right)}{G\left(z_{1}\right)} \leq \frac{1}{\gamma} .
$$

It follows that

$$
\begin{aligned}
G\left(z_{1}\right) & =\Upsilon\left(\frac{1}{\Upsilon}+\frac{1}{\gamma}\right) G\left(z_{1}\right)-\Upsilon H\left(z_{1}\right)=\frac{\Upsilon(\Upsilon+\gamma) G\left(z_{1}\right)-\Upsilon^{2} \gamma H\left(z_{1}\right)}{\gamma \Upsilon}=\left(\frac{\Upsilon}{\gamma}+1\right) G\left(z_{1}\right)-\Upsilon H\left(z_{1}\right) \\
& \leq \Upsilon\left[\left(\frac{\Upsilon}{\gamma}+1\right) G\left(z_{1}\right)-\Upsilon H\left(z_{1}\right)\right] \leq \Delta(G(\varphi), H(\varphi)) .
\end{aligned}
$$

From (80) and (85), we clearly see that

$$
\begin{aligned}
& G^{v}\left(z_{1}\right) \leq \Delta^{v}(G(\varphi), H(\varphi)), \\
& H^{v}\left(z_{1}\right) \leq \Delta^{v}(G(\varphi), H(\varphi)) .
\end{aligned}
$$

$$
\frac{1}{\sigma^{\delta} \Gamma(\delta)} \frac{\exp \left[((\sigma-1) / \sigma)\left(\Phi(\varphi)-\Phi\left(z_{1}\right)\right)\right] \Phi^{\prime}\left(z_{1}\right)}{\left(\Phi(\varphi)-\Phi\left(z_{1}\right)\right)^{1-\delta}}
$$

Multiplying both sides of (86) with

$$
\begin{gathered}
\frac{1}{\sigma^{\delta} \Gamma(\delta)} \int_{x}^{\varphi} \frac{\exp \left[((\sigma-1) / \sigma)\left(\Phi(\varphi)-\Phi\left(z_{1}\right)\right)\right] \Phi^{\prime}\left(z_{1}\right)}{\left(\Phi(\varphi)-\Phi\left(z_{1}\right)\right)^{1-\delta}} G^{v}\left(z_{1}\right) \mathrm{d} z_{1} \leq \frac{1}{\sigma^{\delta} \Gamma(\delta)} \\
\int_{x}^{\varphi} \frac{\exp \left[((\sigma-1) / \sigma)\left(\Phi(\varphi)-\Phi\left(z_{1}\right)\right)\right] \Phi^{\prime}\left(z_{1}\right)}{\left(\Phi(\varphi)-\Phi\left(z_{1}\right)\right)^{1-\delta}} \Delta^{v}(G(\varphi), H(\varphi)) \mathrm{d} z_{1} .
\end{gathered}
$$

Inequality (89) can be written as

$$
\left(\mathscr{T}_{x, \Phi}^{\delta, \sigma} G^{v}(\varphi)\right)^{1 / v} \leq\left(\mathscr{T}_{x, \Phi}^{\delta, \sigma} \Delta^{v}(G(\varphi), H(\varphi))\right)^{1 / v} .
$$

Similary, from (87), we obtain

$$
\left(\mathscr{T}_{x, \Phi}^{\delta, \sigma} H^{v}(\varphi)\right)^{1 / v} \leq\left(\mathscr{T}_{x, \Phi}^{\delta, \sigma} \Delta^{v}(G(\varphi), H(\varphi))\right)^{1 / v}
$$

Therefore, inequality (76) follows easily from (90) and (91).

\section{Conclusion}

In this paper, we introduce a nonlocal generalized proportional fractional integral operator with respect to another function $\Phi$, and then we derived several variants concerning to the reverse Minkowski inequality by involving the generalized proportional fractional integral operator with respect to another function $\Phi$; as a particular case, the inequality involving fractional integrals in the 
Riemann-Liouville, Hadamard, and Katugampola sense can be found by choosing appropriate and suitable substitutions in the proportionality index $\sigma$ and $\Phi$. The variants obtained in this research will lead to the inequalities which are established earlier by Rahman et al. [47] and numerous outcomes can be generalized for the application of these newly introduced fractional integral operators by utilizing Remark 1. Note that the outcomes in this paper are like hypothetically surely understood proliferation properties of fractional Schrödinger equation $[55,56]$. Besides, our outcomes are practically identical to equality-time evenness in a fractional Schrödinger equation [57]. Indeed, the work established in the given arrangement is new and contributes suggestively to the study of integrodifferential and difference equations.

\section{Data Availability}

No data were used to support this study.

\section{Conflicts of Interest}

The authors declare that they have no conflicts of interest.

\section{Acknowledgments}

The research was supported by the Natural Science Foundation of China (Grant nos. 11701176, 61673169, 11301127, 11626101, and 11601485).

\section{References}

[1] T. Abdeljawad, "Dual identities in fractional difference calculus within Riemann," Advances in Difference Equations, vol. 2013, no. 1, p. 36, 2013.

[2] T. Abdeljawad, "On conformable fractional calculus," Journal of Computational and Applied Mathematics, vol. 279, pp. 57-66, 2015.

[3] K. S. Miller and B. Ross, An Introduction to the Fractional Calculus and Fractional Differential Equations, John Wiley \& Sons, New York, NY, USA, 1993.

[4] S. G. Samko, A. A. Kilbas, and O. I. Marichev, Fractional Integrals and Derivatives, Gordon and Breach Science Publishers, Yverdon, Switzerland, 1993.

[5] V. Kiryakova, Generalized Fractional Calculus and Applications, Longman Scientific \& Technical, Harlow, UK, 1994.

[6] D. Baleanu, K. Diethelm, E. Scalas, and J. J. Trujillo, Fractional Calculus: Models and Numerical Methods, World Scientific Publishing, Hackensack, NJ, USA, 2012.

[7] R. Almeida, "A Caputo fractional derivative of a function with respect to another function," Communications in Nonlinear Science and Numerical Simulation, vol. 44, pp. 460-481, 2017.

[8] A. A. Kilbas, H. M. Srivastava, and J. J. Trujillo, Theory and Applications of Fractional Differential Equations, Elsevier Science B.V., Amsterdam, Netherlands, 2006.

[9] S. Rashid, F. Jarad, M. A. Noor, H. Kalsoom, and Y.-M. Chu, "Inequalities by means of generalized proportional fractional integral operators with respect to another function," Mathematics, vol. 7, no. 12, p. 1225, 2020.

[10] F. M. Atici and P. W. Eloe, "Initial value problems in discrete fractional calculus," Proceedings of the American Mathematical Society, vol. 137, no. 3, pp. 981-989, 2009.
[11] H. L. Gray and N. F. Zhang, "On a new definition of the fractional difference," Mathematics of Computation, vol. 50, no. 182, pp. 513-529, 1988.

[12] R. Khalil, M. Al Horani, A. Yousef, and M. Sababheh, "A new definition of fractional derivative," Journal of Computational and Applied Mathematics, vol. 264, pp. 65-70, 2014.

[13] S. Rashid, M. A. Noor, K. I. Noor, F. Safdar, and Y.-M. Chu, "Hermite-Hadamard type inequalities for the class of convex functions on time scale," Mathematics, vol. 7, no. 10, p. 956, 2019.

[14] S. Rashid, F. Jarad, H. Kalsoom, and Y.-M. Chu, "On PólyaSzegö and Čebyšev type inequalites via generalized $k$-fractional integrals," Adveances in Difference Equations, vol. 2020, no. 1, p. 125, 2020.

[15] S. Rafeeq, H. Kalsoom, S. Hussain, S. Rashid, and Y.-M. Chu, "Delay dymanic double inegral inequalities on time scales with applications," Adveances in Difference Equations, vol. 2020, p. 40, 2020.

[16] S. Rashid, M. A. Noor, K. I. Noor et al., "Ostrowski type inequalities in the sense of generalized $\mathrm{K}$-fractional integral operator for exponentially convex functions," AIMS Mathematics, vol. 5, no. 3, pp. 2629-2645, 2020.

[17] S. Khan, M. Adil Khan, and Y. M. Chu, "Converses of the Jensen inequality derived from the Green functions with applications in information theory," Mathematical Methods in the Applied Sciences, vol. 43, no. 5, pp. 2577-2587, 2020.

[18] M. Adil Khan, N. Mohammad, E. R. Nwaeze, and Y.-M. Chu, "Quantum Hermite-Hadamard inequality by means of a green function," Adveances in Difference Equations, vol. 2020, no. 1, p. 99, 2020.

[19] T.-H. Zhao, L. Shi, and Y.-M. Chu, "Convexity and concavity of the modified Bessel functions of the first kind with respect to Hölder means," Revista de la Real Academia de Ciencias Exactas, Físicas y Naturales. Serie A. Matemáticas, vol. 114, no. 2, 2020.

[20] M.-K. Wang, Z.-Y. He, and Y.-M. Chu, "Sharp power mean inequalities for the generalized elliptic integral of the first kind," Computational Methods and Function Theory, vol. 20, no. 1, pp. 111-124, 2020.

[21] M. Wang, W. Zhang, and Y. Chu, "Monotonicity, convexity and inequalities involving the generalized elliptic integrals," Acta Mathematica Scientia, vol. 39, no. 5, pp. 1440-1450, 2019.

[22] T.-R. Huang, S.-Y. Tan, X.-Y. Ma, and Y.-M. Chu, "Monotonicity properties and bounds for the complete p-elliptic integrals," Journal of Inequalities and Applications, vol. 2018, p. 239, 2018.

[23] I. Abbas Baloch and Y.-M. Chu, "Petrović-type inequalities for harmonic $h$-convex functions," Journal of Function Spaces, vol. 2020, Article ID 3075390, 7 pages, 2020.

[24] M. Adil Khan, Y. Khurshid, T.-S. Du, and Y.-M. Chu, "Generalization of Hermite-Hadamard type inequalities via conformable fractional integrals," Journal of Function Spaces, vol. 2018, Article ID 5357463, 12 pages, 2018.

[25] A. Iqbal, M. Adil Khan, S. Ullah, and Y.-M. Chu, "Some new Hermite-Hadamard-type inequalities associated with conformable fractional integrals and their applications," Journal of Function Spaces, vol. 2020, Article ID 9845407, 18 pages, 2020.

[26] H.-J. Hu, T.-S. Yi, and X.-F. Zou, "On spatial-temporal dynamics of a Fisher-KPP equation with a shifting environment," Proceedings of the American Mathematical Society, vol. 148, no. 1, pp. 213-221, 2020.

[27] C. Huang, S. Guo, and L. Liu, "Boundedness on Morrey space for Toeplitz type operator associated to singular integral 
operator with variable Calderón-Zygmund kernel," Journal of Mathematical Inequalities, vol. 8, no. 3, pp. 453-464, 2014.

[28] F. Liu, L. Feng, V. Anh, and J. Li, "Unstructured-mesh Galerkin finite element method for the two-dimensional multi-term time-space fractional Bloch-Torrey equations on irregular convex domains," Computers \& Mathematics with Applications, vol. 78, no. 5, pp. 1637-1650, 2019.

[29] C. Huang, H. Zhang, and L. Huang, "Almost periodicity analysis for a delayed Nicholson's blowflies model with nonlinear density-dependent mortality term," Communications on Pure \& Applied Analysis, vol. 18, no. 6, pp. 3337-3349, 2019.

[30] J. Wang, C. Huang, and L. Huang, "Discontinuity-induced limit cycles in a general planar piecewise linear system of saddle-focus type," Nonlinear Analysis: Hybrid Systems, vol. 33, pp. 162-178, 2019.

[31] W.-M. Qian, Z.-Y. He, H.-W. Zhang, and Y.-M. Chu, "Sharp bounds for Neuman means in terms of two-parameter contraharmonic and arithmetic mean," Journal of Inequalities and Applications, vol. 2019, p. 168, 2019.

[32] Y. Jiang and $\mathrm{X}$. $\mathrm{Xu}$, "A monotone finite volume method for time fractional Fokker-Planck equations," Science China Mathematics, vol. 62, no. 4, pp. 783-794, 2019.

[33] J. Wang, X. Chen, and L. Huang, "The number and stability of limit cycles for planar piecewise linear systems of node-saddle type," Journal of Mathematical Analysis and Applications, vol. 469, no. 1, pp. 405-427, 2019.

[34] R. P. Agarwal and A. Özbekler, "Lyapunov type inequalities for mixed nonlinear Riemann-Liouville fractional differential equations with a forcing term," Journal of Computational and Applied Mathematics, vol. 314, pp. 69-78, 2017.

[35] D. D. Baïnov and P. S. Simeonov, Integral Inequalities and Applications, Kluwer Academic Publishers Group, Dordrecht, Netherlands, 1992.

[36] L. Bougoffa, "On Minkowski and Hardy integral inequalities," Journal of Inequalities in Pure and Applied Mathematics, vol. 7, no. 2, p. 60, 2006.

[37] Z.-H. Yang, W.-M. Qian, W. Zhang, and Y. -M. Chu, "Notes on the complete elliptic integral of the first kind," Mathematical Inequalities \& Applications, vol. 23, no. 1, pp. 77-93, 2020.

[38] M.-K. Wang, M.-Y. Hong, Y.-F. Xu, Z.-H. Shen, and Y.-M. Chu, "Inequalities for generalized trigonometric and hyperbolic functions with one parameter," Journal of Mathematical Inequalities, vol. 14, no. 1, pp. 1-21, 2020.

[39] Y. Tan, C. Huang, B. Sun, and T. Wang, "Dynamics of a class of delayed reaction-diffusion systems with Neumann boundary condition," Journal of Mathematical Analysis and Applications, vol. 458, no. 2, pp. 1115-1130, 2018.

[40] C. Huang and L. Liu, "Boundedness of multilinear singular integral operator with non-smooth kernels and mean oscillation," Quaestiones Mathematicae, vol. 40, no. 3, pp. 295-312, 2017.

[41] L. Duan and C. Huang, "Existence and global attractivity of almost periodic solutions for a delayed differential neoclassical growth model," Mathematical Methods in the Applied Sciences, vol. 40, no. 3, pp. 814-822, 2017.

[42] C. Huang, Z. Yang, T. Yi, and X. Zou, "On the basins of attraction for a class of delay differential equations with nonmonotone bistable nonlinearities," Journal of Differential Equations, vol. 256, no. 7, pp. 2101-2114, 2014.

[43] Z. Dahmani, "On Minkowski and Hermite-Hadamard integral inequalities via fractional integration," Annals of Functional Analysis, vol. 1, no. 1, pp. 51-58, 2020.
[44] A. Anber, Z. Dahmani, and B. Bendoukha, "New integral inequalities of Feng Qi type via Riemann-Liouville fractional integration," Facta Universitatis, Series: Mathematics and Informations, vol. 27, no. 2, pp. 157-166, 2012.

[45] J. Vanterler da Costa Sousa and E. Capelas de Oliveira, "The Minkowski's inequality by means of a generalized fractional integral," AIMS Mathematics, vol. 3, no. 1, pp. 131-147, 2018.

[46] S. Mubeen, S. Habib, and M. N. Naeem, "The Minkowski inequality involving generalized $k$-fractional conformable integral," Journal of Inequalities and Applications, vol. 2019, p. 81, 2019.

[47] G. Rahman, A. Khan, T. Abdeljawad, and K. S. Nisar, "The Minkowski inequalities via generalized proportional fractional integral operators," Advances in Difference Equations, vol. 2019, p. 287, 2019.

[48] S. Taf and K. Brahim, "Some new results using Hadamard fractional integral," International Journal of Nonlinear Analysis and Applications, vol. 7, no. 1, pp. 103-109, 2016.

[49] E. Set, M. E. Özdemir, and S. S. Dragomir, "On the HermiteHadamard inequality and other integral inequalities involving two functions," Journal of Inequalities and Applications, vol. 2010, Article ID 148102, 9 pages, 2010.

[50] T.-H. Zhao, Y.-M. Chu, and H. Wang, "Logarithmically complete monotonicity properties relating to the gamma function," Abstract and Applied Analysis, vol. 2011, Article ID 896483, 13 pages, 2011.

[51] Z.-H. Yang, W.-M. Qian, Y.-M. Chu, and W. Zhang, "On rational bounds for the gamma function," Journal of Inequalities and Applications, vol. 2017, p. 210, 2017.

[52] T.-R. Huang, B.-W. Han, X.-Y. Ma, and Y.-M. Chu, "Optimal bounds for the generalized Euler-Mascheroni constant," Journal of Inequalities and Applications, vol. 2018, p. 118, 2018.

[53] F. Jarad, T. Abdeljawad, and J. Alzabut, "Generalized fractional derivatives generated by a class of local proportional derivatives," The European Physical Journal Special Topics, vol. 226, no. 16-18, pp. 3457-3471, 2017.

[54] G. Rahman, T. Abdeljawad, F. Jarad, A. Khan, and K. S. Nisar, "Certain inequalities via generalized proportional Hadamard fractional integral operators," Advances in Difference Equations, vol. 2019, p. 454, 2019.

[55] D. Zhang, Y.-Q. Zhang, Z.-Y. Zhang et al., "Unveiling the link between fractional Schrödinger equation and light propagation in honeycomb lattice," Annalen der Physik, vol. 529, no. 9, Article ID 1700149, 2017.

[56] Y. Zhang, R. Wang, H. Zhong, J. Zhang, M. R. Belić, and Y. Zhang, "Resonant mode conversions and Rabi oscillations in a fractional Schrödinger equation," Optics Express, vol. 25, no. 26, pp. 32401-32410, 2017.

[57] Y. Zhang, H. Zhong, M. R. Belić et al., "PT symmetry in a fractional Schrödinger equation," Laser \& Photonics Reviews, vol. 10, no. 3, pp. 526-531, 2016. 